\title{
6S RNA regulation of relA alters ppGpp levels in early stationary phase
}

\author{
Correspondence \\ Karen M. Wassarman \\ wassarman@bact.wisc.edu
}

Received 21 July 2010

Revised 3 September 2010

Accepted 7 September 2010

\author{
Amy T. Cavanagh, Pete Chandrangsu and Karen M. Wassarman \\ Department of Bacteriology, University of Wisconsin-Madison, 1550 Linden Dr., Madison, \\ WI 53706, USA
}

6S RNA is a small, non-coding RNA that interacts directly with $\sigma^{70}$-RNA polymerase and regulates transcription at many $\sigma^{70}$-dependent promoters. Here, we demonstrate that $6 S$ RNA regulates transcription of relA, which encodes a ppGpp synthase. The 6S RNA-dependent regulation of relA expression results in increased ppGpp levels during early stationary phase in cells lacking 6S RNA. These changes in ppGpp levels, although modest, are sufficient to result in altered regulation of transcription from $\sigma^{70}$-dependent promoters sensitive to ppGpp, including those promoting expression of genes involved in amino acid biosynthesis and rRNA. These data place 6S RNA as another player in maintaining appropriate gene expression as cells transition into stationary phase. Independent of this ppGpp-mediated 6S RNA-dependent regulation, we also demonstrate that in later stationary phase, 6S RNA continues to downregulate transcription in general, and specifically at a subset of the amino acid promoters, but through a mechanism that is independent of ppGpp and which we hypothesize is through direct regulation. In addition, 65 RNA-dependent regulation of $\sigma^{S}$ activity is not mediated through observed changes in ppGpp levels. We suggest a role for $6 \mathrm{~S}$ RNA in modulating transcription of several global regulators directly, including relA, to downregulate expression of key pathways in response to changing environmental conditions.

\section{INTRODUCTION}

6S RNA is an untranslated, small RNA that was first discovered in Escherichia coli as a highly abundant RNA (Hindley, 1967). The cellular function of 6S RNA remained elusive for many years, but it is now known that it regulates gene expression through direct interaction with $\sigma^{70}$-RNA polymerase $\left(\sigma^{70}\right.$-RNAP) (for reviews, see Willkomm \& Hartmann, 2005; Wassarman, 2007). Although 6S RNA is present throughout growth, it accumulates to its highest levels during late stationary phase, when most $\mathrm{E} \sigma^{70}$ is bound by 6 S RNA (Wassarman \& Storz, 2000).

Intriguingly, even during late stationary phase, 6S RNAdependent downregulation of $\sigma^{70}$-dependent transcription is promoter-specific (Trotochaud \& Wassarman, 2004). $\sigma^{70}$-Dependent promoters are primarily recognized through two core sequences: the -10 element (consensus TATAAT) and the -35 element (consensus TTGATA). In addition, a subset of promoters contains what is referred to as an extended -10 element (consensus TGnTATAAT), which is defined by an additional conserved TG $1 \mathrm{bp}$ upstream of the -10 element (Voskuil et al., 1995; Bown et al., 1997). Previously, we determined that an extended -10 element and a -35 element with a weak match to the

Abbreviations: $\sigma^{70}$-RNAP, $\sigma^{70}$-RNA polymerase; CRP, cAMP receptor protein. consensus are two features that independently contribute to the 6S RNA sensitivity of promoter expression during late stationary phase (Cavanagh et al., 2008).

To date, studies on 6S RNA regulation of transcription have focused primarily on late stationary phase $(>18 \mathrm{~h}$ of growth in rich medium) when 6S RNA levels are maximal, although it also has been shown that the presence of $6 \mathrm{~S}$ RNA leads to altered transcription during late exponential and early stationary phase (Trotochaud \& Wassarman, 2004, 2006; Neusser et al., 2010). Identified phenotypes associated with the loss of $6 \mathrm{~S}$ RNA are most prominent in late and extended stationary phase, and include altered cell survival, particularly during competitive growth and growth at high $\mathrm{pH}$ (Trotochaud \& Wassarman, 2004, 2006).

During the transition from exponential to stationary phase, a large number of regulators are involved in altering gene expression to facilitate cell adaptation and survival in a changing environment lacking optimal nutrients. One molecule important during this transition is ppGpp (Paul et al., 2004; Gralla, 2005; Magnusson et al., 2005; Potrykus \& Cashel, 2008; Srivatsan \& Wang, 2008). Decreasing availability of amino acids is largely sensed by RelA, a ppGpp synthase I that is associated with ribosomes. As uncharged tRNAs accumulate as a result of decreasing amino acid pools, RelA is activated and synthesizes ppGpp. 
In turn, ppGpp binds to RNA polymerase and regulates transcription at ppGpp-sensitive promoters. Many of the promoters that are upregulated by ppGpp direct the expression of genes involved in amino acid biosynthesis and uptake (Barker et al., 2001). Conversely, the rRNA genes are downregulated by ppGpp. In this manner, ppGpp is a key player in the response to declining nutrients and in the control of growth rate through regulation of ribosome synthesis.

Here, we demonstrate that 6S RNA regulates the expression of $r e l A$, which leads to changes in ppGpp levels during early stationary phase, and thus adds another layer of regulation to the complex network of responses that occur as cells transition to less favourable growth conditions. We show that 6S RNA-dependent changes in ppGpp levels during early stationary phase (i.e. $6 \mathrm{~h}$ of growth) are sufficient to lead to changes in the transcription of target genes such as those involved in amino acid biosynthesis and rRNA. Therefore, 6S RNA regulates transcription during this time frame in spite of being at subsaturating levels for binding to $\sigma^{70}$-RNAP. We also demonstrate that 6S RNA-dependent changes in transcription in late stationary phase $(>24 \mathrm{~h})$ are independent of ppGpp, thus revealing the diversity of mechanisms for 6S RNA-dependent regulation of gene expression during different times of cell growth and survival.

\section{METHODS}

Strains. E. coli strains (Table 1) were grown in EZ Rich Defined Medium [RDM; a MOPS-based medium (Neidhardt et al., 1974) supplemented with $0.2 \%$ glucose, 20 amino acids, vitamins and nucleobases] (Teknova) at $37{ }^{\circ} \mathrm{C}$ unless indicated otherwise. Promoter-lac $Z$ fusions were all chromosomal as $\lambda$-phage lysogens, and were generated as described by Rao et al. (1994). Promoter regions for $\operatorname{relAP} 1_{(-100+50)}$ and relAP2 $(-100+50)$ were generated by PCR amplification from genomic DNA with oligonucleotides, and relAP $1_{(-42+2)}$ and relAP2 $2_{(-41+2)}$ by annealing oligonucleotides containing the entire promoter region with appropriate overhangs. Promoter regions were cloned into the EcoRI and HindIII sites in pMSB1 (Rao et al., 1994). The relA promoter sequences are shown in Fig. 1(a); full oligonucleotide sequences are available upon request. The nomenclature for all promoter-lac $Z$ fusions indicates the extent of promoter regions included in the reporter as the number of nucleotides upstream and downstream of the +1 transcription start site (see Table 1). All plasmid intermediates and the chromosomal regions containing the lysogen promoters were confirmed by sequencing. Promoter P1 transductions were performed as described by Silhavy et al. (1984) to move ssrS1 (Lee et al., 1985) and $\Delta$ relA (relA251::kan; Xiao et al., 1991) into various strain backgrounds as needed.

The ssrS1 allele contains a bla insertion into the 6S RNA gene (ssrS) (Lee et al., 1985). Alleles with a precise replacement of the 6S RNA coding sequence with a tetracycline-resistance cassette (ssrS2) or a $13 \mathrm{bp}$ scar after Flp-mediated removal of the tetracycline-resistance cassette (ssrS3) have been described previously (Trotochaud \& Wassarman, 2006). For all three alleles (ssrS1, ssrS2 and ssrS3), no detectable 6S RNA is expressed, and expression of the downstream gene $(y g f A)$ is the same as in the wild-type, indicating that they are non-polar on $y g f A$, and the $\beta$-galactosidase activity of reporter genes and growth phenotypes are indistinguishable (Trotochaud \& Wassarman, 2006; Cavanagh et al., 2008).

For the microarray experiment, RNA was isolated from two independent cultures each of wild-type (MG1655; KW489) or 6S RNA null (ssrS3; KW490) cells grown at $37{ }^{\circ} \mathrm{C}$ for $16 \mathrm{~h}$ after dilution to $\mathrm{OD}_{600} 0.05$ into RDM. RNA samples were sent to Nimblegen for analysis using the standard protocol for measuring mRNA levels on $E$. coli expression microarrays (Design T183333 60-mer, E. coli K-12). The full dataset will be published elsewhere (A. T. Cavanagh \& K. M. Wassarman, unpublished data).

ppGpp analysis. Levels of ppGpp were measured after formic acid extraction from cells as described by Schneider et al. (2003). In short, overnight cultures grown in $\mathrm{RDM}$ at $37{ }^{\circ} \mathrm{C}$ were diluted to $\mathrm{OD}_{600} 0.1$ into $2 \mathrm{ml} \mathrm{RDM}$ containing $1 \mathrm{mM} \mathrm{K}_{2} \mathrm{HPO}_{4}$ and $40 \mu \mathrm{Ci}(1.48 \mathrm{MBq})$ $\left[{ }^{32} \mathrm{P}\right]$ orthophosphoric acid (PerkinElmer) and further incubated at $37{ }^{\circ} \mathrm{C}$ for the times indicated. For nucleotide extraction, $200 \mu \mathrm{l}$ cell culture was added to $40 \mu \mathrm{l}$ cold $2 \mathrm{M}$ formic acid and incubated on ice for $20 \mathrm{~min}$. Following centrifugation, $20 \mu \mathrm{l}$ extract was spotted onto a polyethylenimine (PEI)-cellulose F TLC plate (EMD Chemicals) and separated in $0.85 \mathrm{M} \mathrm{KH}_{2} \mathrm{PO}_{4}(\mathrm{pH} 3.4)$. ppGpp was identified by migration profile as well as by its absence in $\Delta$ relA $\Delta$ spoT strain backgrounds, and the level of ppGpp was quantified on a Typhoon phosphorimager. Note that previous discrepancies concerning nucleotide isolation and quantification primarily resulted from formaldehyde-based isolation methodologies (Schneider \& Gourse, 2004). Therefore, formic acid extraction was used here, which allows comparison of ppGpp levels between cell types over time.

$\boldsymbol{\beta}$-Galactosidase assays. $\beta$-Galactosidase activity was measured at $30{ }^{\circ} \mathrm{C}$ as previously described (Trotochaud \& Wassarman, 2004), and activity is expressed in Miller units $\left(\Delta \mathrm{OD}_{420} \mathrm{~min}^{-1}\right.$ per $\mathrm{OD}_{600}$ unit) (Miller, 1972). Briefly, cultures inoculated from a single colony were grown for $18 \mathrm{~h}$ in RDM, diluted approximately $1: 100$ in fresh medium (to $\mathrm{OD}_{600} 0.04$ ) and grown for $24 \mathrm{~h}$, at which time they were analysed (24 h time point) or rediluted into fresh medium (1:100) and grown for an additional $6 \mathrm{~h}$ prior to analysis $(6 \mathrm{~h}$ time point). Cell growth was at $37{ }^{\circ} \mathrm{C}$ for all strain backgrounds except those containing the hisG-lacZ reporter, which was at $30{ }^{\circ} \mathrm{C}$ as it is a 'system I' lysogen that is temperature-sensitive (Rao et al., 1994). For $\beta$-galactosidase assays, cells were lysed with SDS and chloroform. At least three independent cultures per strain were used per experiment, and experiments were repeated at least three times.

\section{RESULTS}

Microarray expression analysis comparing mRNA levels between wild-type and 6S RNA null cells (ssrS3) demonstrated that expression of many genes was altered in a $6 \mathrm{~S}$ RNA-dependent manner (A. T. Cavanagh \& K. M. Wassarman, unpublished data). One mRNA that increased in the absence of 6S RNA was relA, which encodes ppGpp synthase I. Specifically, in cells lacking 6S RNA, relA mRNA was increased 2.3-fold relative to wild-type cells in late stationary phase ( $16 \mathrm{~h}$ of growth in RDM). Given the importance of ppGpp in regulating gene expression in response to nutrient limitation, a time when 6S RNA function is also important, we examined the relationship between 6S RNA function, relA expression and ppGpp levels. Expression of spoT, which encodes the bifunctional SpoT enzyme that can synthesize and degrade ppGpp (Magnusson et al., 2005; Potrykus \& Cashel, 2008), was 
Table 1. Bacterial strains used in this study

\begin{tabular}{|c|c|c|}
\hline Strain & Genotype & Reference or source \\
\hline KW489 & E. coli $\mathrm{K}-12 \mathrm{MG} 1655$ & \\
\hline KW490 & KW489 ssrS3 & Cavanagh et al. (2008) \\
\hline RLG3499 & MG1655 pyrE + lacI lacZ [VH1000] & Gaal et al. (2001) \\
\hline KW348 & RLG3499 ssrS1 & Trotochaud \& Wassarman (2006) \\
\hline RLG10110 & RLG3499 $\lambda$ relAP1 $(-100+50)-$ lacZ & This work \\
\hline KW702 & RLG10110 ssrS1 & This work \\
\hline RLG10111 & RLG3499 $\lambda$ relAP2 $(-100+50)-l a c Z$ & This work \\
\hline KW703 & RLG10111 ssrS1 & This work \\
\hline KW704 & RLG3499 $\lambda$ relAP1 $(-41+2)-l a c Z$ & This work \\
\hline KW705 & KW704 ssrS1 & This work \\
\hline KW706 & KW704 relA251:: $\operatorname{kan}^{\mathrm{r}}$ & This work \\
\hline KW707 & KW704 ssrS1 relA251:: $\operatorname{kan}^{\mathrm{r}}$ & This work \\
\hline KW708 & RLG3499 $\lambda$ relAP2 $(-42+2)-l a c Z$ & This work \\
\hline KW709 & KW708 ssrS1 & This work \\
\hline KW710 & KW708 relA251:: $\operatorname{kan}^{\mathrm{r}}$ & This work \\
\hline KW711 & KW708 ssrS1 relA251:: $\operatorname{kan}^{\mathrm{r}}$ & This work \\
\hline RLG4418 & RLG3499 $\lambda h i s G(-60+15)-l a c Z$ & Barker et al. (2001) \\
\hline KW359 & RLG4418 ssrS1 & Cavanagh et al. (2008) \\
\hline KW712 & RLG4418 relA251:: $\operatorname{kan}^{\mathrm{r}}$ & This work \\
\hline KW713 & RLG4418 ssrS1 relA251:: $\mathrm{kan}^{\mathrm{r}}$ & This work \\
\hline RLG5080 & RLG3499 $\lambda$ thrABC(-72+1)-lacZ & Barker et al. (2001) \\
\hline KW459 & RLG5080 ssrS1 & Cavanagh et al. (2008) \\
\hline KW714 & RLG5080 relA251:: $\operatorname{kan}^{\mathrm{r}}$ & This work \\
\hline KW715 & RLG5080 ssrS1 relA251:: $\mathrm{kan}^{\mathrm{r}}$ & This work \\
\hline RLG4422 & RLG3499 $\lambda \operatorname{livJ}(-60+13)-\operatorname{lac} Z$ & Barker et al. (2001) \\
\hline KW464 & RLG4422 ssrS1 & Cavanagh et al. (2008) \\
\hline KW716 & RLG4422 relA251:: $\operatorname{kan}^{\mathrm{r}}$ & This work \\
\hline KW717 & RLG4422 ssrS1 relA251:: $\operatorname{kan}^{\mathrm{r}}$ & This work \\
\hline RLG4818 & RLG3499 $\lambda$ pheA $(-73+10)-$ lacZ & Barker et al. (2001) \\
\hline KW351 & RLG4818 ssrS1 & Cavanagh et al. (2008) \\
\hline KW718 & RLG4818 relA251:: $\operatorname{kan}^{\mathrm{r}}$ & This work \\
\hline KW719 & RLG4818 ssrS1 relA251:: $\operatorname{kan}^{\mathrm{r}}$ & This work \\
\hline RLG6358 & RLG3499 $\lambda \operatorname{rrnBP} 1(-41+1)-l a c Z$ & Hirvonen et al. (2001) \\
\hline KW238 & RLG6358 ssrS1 & Trotochaud \& Wassarman (2004) \\
\hline KW720 & RLG6358 relA251:: $\operatorname{kan}^{\mathrm{r}}$ & This work \\
\hline KW721 & RLG6358 ssrS1 relA251:: $\operatorname{kan}^{\mathrm{r}}$ & This work \\
\hline KW460 & RLG3499 $\lambda \operatorname{lacUV5}(-48+4)-l a c Z$ & Cavanagh et al. (2008) \\
\hline KW461 & KW460 ssrS1 & Cavanagh et al. (2008) \\
\hline KW722 & KW460 relA251:: $\operatorname{kan}^{\mathrm{r}}$ & This work \\
\hline KW723 & KW460 ssrS1 relA251:: $\operatorname{kan}^{\mathrm{r}}$ & This work \\
\hline RLG3760 & RLG3499 $\lambda$ bolA1 $(-54+16)-$ lacZ & Gaal et al. (2001) \\
\hline KW378 & RLG3760 ssrS1 & Trotochaud \& Wassarman (2004) \\
\hline KW724 & RLG3760 relA251:: $\operatorname{kan}^{\mathrm{r}}$ & This work \\
\hline KW725 & RLG3760 ssrS1 relA251:: $\operatorname{kan}^{\mathrm{r}}$ & This work \\
\hline
\end{tabular}

relatively unchanged (0.85 in cells lacking $6 \mathrm{~S}$ RNA compared with wild-type cells).

\section{S RNA regulates transcription of relA}

relA expression is driven by two identified promoters, P1 and P2 (see Fig. 1a) (Metzger et al., 1988; Nakagawa et al., 2006). relAP1, located 178 bp upstream of the translational start site for $\mathrm{relA}$, is constitutively active; relAP2, located $626 \mathrm{bp}$ upstream of $\mathrm{rel} A$, is induced during the transition into stationary phase (Metzger et al., 1988; Nakagawa et al.,
2006). Interestingly, relAP1 has a fairly weak -35 element when compared with the consensus (TgGAac) and relAP2 contains an extended -10 element; both these features are independent indicators of promoters sensitive to 6S RNA (Cavanagh et al., 2008), and therefore we predicted that both relAP1 and relAP2 would be directly downregulated by $6 \mathrm{~S}$ RNA. To test whether these promoters respond to $6 \mathrm{~S}$ RNA regulation, promoter-lac $Z$ fusions were generated to allow expression to be monitored by $\beta$-galactosidase activity in cells with 6S RNA or lacking 6S RNA (ssrS1). In agreement with our predictions, both relAP1 $(-100+50)$ 
(a)
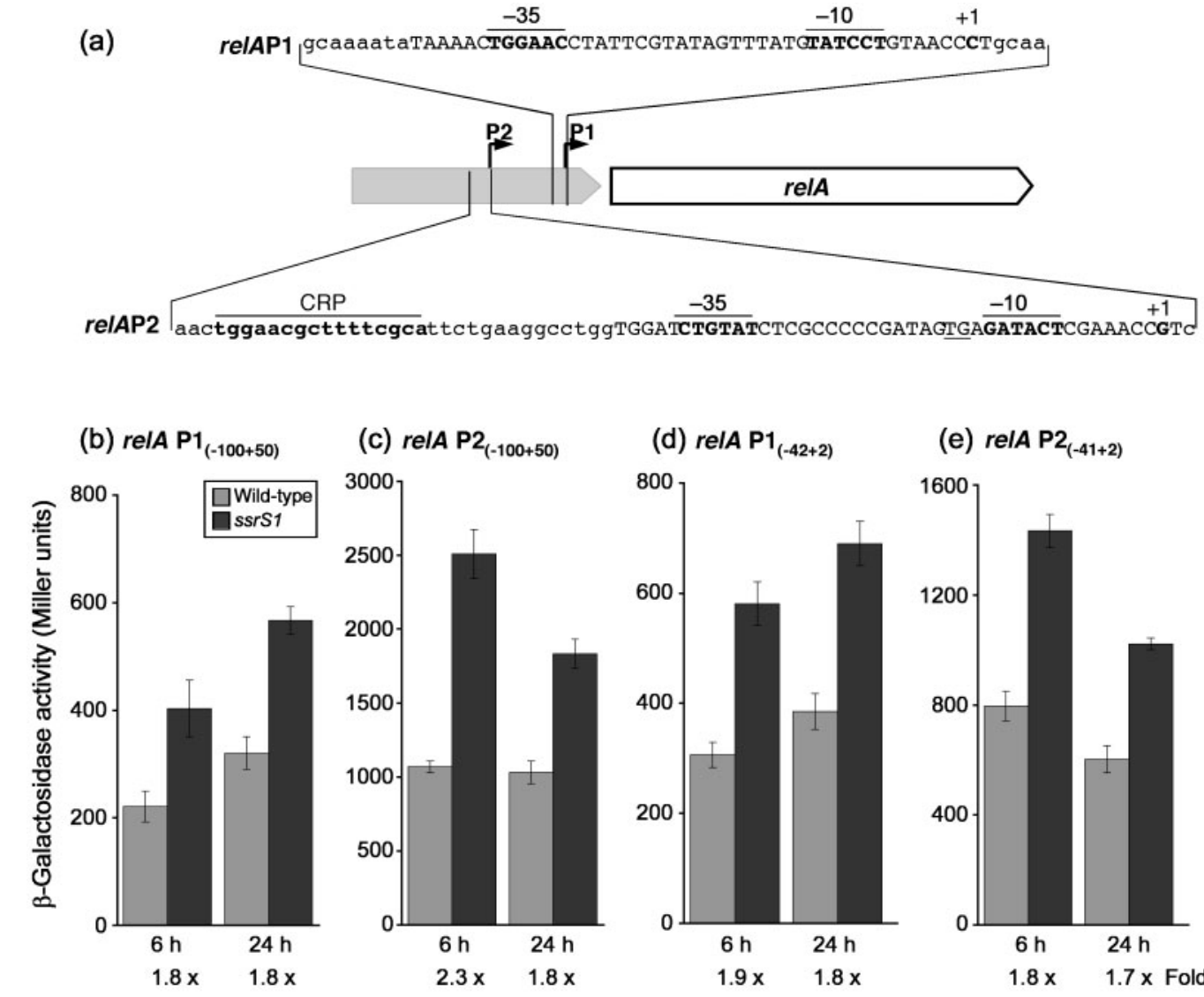

(e) relA P2 ${ }_{(-41+2)}$

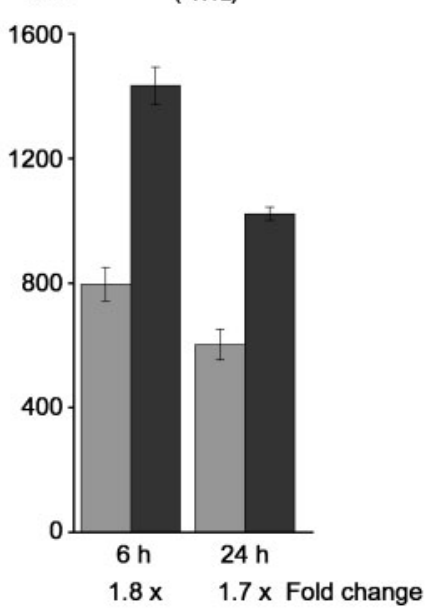

Fig. 1. relA $\mathrm{P} 1$ and $\mathrm{P} 2$ promoters are both sensitive to 6S RNA. (a) Schematic of relA (white box) and its genomic location. Promoters $\mathrm{P} 1$ and $\mathrm{P} 2$ are indicated by arrows, and the sequence of each promoter region is shown above (P1) or below (P2) the schematic. Note that both $\mathrm{P} 1$ and $\mathrm{P} 2$ are quite distant upstream from the translational start of rel $A$ and are located within the upstream gene $(r / m D)$ represented by a grey box. The start site for transcription $(+1)$, the -10 and -35 elements, and the CRP binding sequence are shown in bold and labelled above the sequences. The TG of the extended -10 element for P2 is underlined. Upper-case type indicates the extent of sequence included in the minimal promoters $\operatorname{rel} / A \mathrm{P} 1_{(-42+2)}$ and relAP2 $(-41+2)$. Note that the full sequence included in relAP1 $(-100+50)$ and $r e / A P 2_{(-100+50)}$ extends beyond the sequences shown. (b-e) $\beta$-Galactosidase activity from relA promoter-lacZ reporters. Wild-type and 6S RNA null (ssrS1) strains containing chromosomal copies of the promoters indicated were grown to late stationary phase $(24 \mathrm{~h})$ in RDM at $37{ }^{\circ} \mathrm{C}$. Fold change is the $\beta$-galactosidase activity in ssrS1 divided by the $\beta$-galactosidase activity in the wild-type background. Data shown are the mean of at least three independent experiments with three duplicate samples per experiment. Error bars, SD.

and relAP2 $(-100+50)$ had increased expression in cells lacking 6S RNA relative to the wild-type (1.8- to 2.3-fold) in both early and late stationary phase (6 and $24 \mathrm{~h}$ of growth in RDM) (Fig. 1b, c, Table 2). For comparison, the microarray data were from cells grown for $16 \mathrm{~h}$ in the same medium. Together, these data suggest that relA mRNA levels are altered in a 6S RNA-dependent manner throughout stationary phase.

The relAP2 promoter contains a cAMP receptor protein (CRP) binding site that is activated by CRP (Nakagawa et al., 2006) and the relAP2 $(-100+50)$ reporter included this CRP site. The microarray data also indicated that crp mRNA levels were increased (1.9-fold) in cells lacking 6S RNA compared with wild-type cells (A. T.
Cavanagh \& K. M. Wassarman, unpublished data). To test whether changes in transcription at relAP2 $(-100+50)$ and relAP1 $1_{(-100+50)}$ were likely to result from direct regulation by $6 \mathrm{~S}$ RNA or might be due to indirect regulation via changes in trans-acting factors such as CRP, we also tested reporters that contained only minimal core promoter sequences lacking known binding sites $\left[\operatorname{relAP}_{(-42+2)}\right.$ and relAP $2_{(-41+2)}$; see Fig. 1a]. The minimal relAP1 $1_{(-42+2)}$ reporter remained sensitive to $6 \mathrm{~S}$ RNA, similar to the longer relAP1 $1_{(-100+50)}$ (compare Fig. $1 \mathrm{~d}$ and Fig. 1b). The minimal relAP2 $(-41+2)$ reporter also remained sensitive to $6 \mathrm{~S}$ RNA, although to a lesser extent than the reporter that also contained the CRP binding site $($ relAP2 $(-100+50)$ (compare Fig. 1e and Fig. 1c). In agreement with CRP activation at this promoter, the overall level of expression 
Table 2. Comparison of promoter activities in cells with and without 6S RNA

Results are given as fold change ( $\beta$-galactosidase activity in ssrS1 divided by $\beta$-galactosidase activity in wild-type cells). Measurements were made with wild-type $\mathrm{relA}\left(\mathrm{rel}^{+}\right)$cells or cells lacking $\mathrm{relA}$ $(\Delta$ relA) grown in RDM for 6 or $24 \mathrm{~h}$ as indicated. For promoter activities and the SD associated with analysis, see Fig. 2.

\begin{tabular}{|c|c|c|c|c|}
\hline \multirow[t]{2}{*}{ Promoter } & \multicolumn{2}{|c|}{$6 \mathrm{~h}$} & \multicolumn{2}{|c|}{$24 \mathrm{~h}$} \\
\hline & $\operatorname{relA} A^{+}$ & $\Delta$ relA & $\operatorname{relA}{ }^{+}$ & $\Delta$ relA \\
\hline hisG & 1.7 & 1.1 & 1.4 & 1.6 \\
\hline thrA & 1.5 & 1.1 & 1.1 & 1.0 \\
\hline livJ & 1.3 & 1.1 & 1.9 & 1.9 \\
\hline pheA & 1.4 & 1.0 & 1.0 & 1.0 \\
\hline$r r n B P 1$ & 0.7 & 1.0 & 1.1 & 1.0 \\
\hline lacUV5 & 0.9 & 0.9 & 1.0 & 1.0 \\
\hline $\operatorname{relAP} 1_{(-42+2)}$ & 1.9 & 1.8 & 1.8 & 1.8 \\
\hline $\operatorname{relAP} 2_{(-41+2)}$ & 1.8 & 1.8 & 1.7 & 1.7 \\
\hline bolA & 0.5 & 0.4 & 0.5 & 0.5 \\
\hline
\end{tabular}

of the minimal relAP2 $2_{(-41+2)}$ was decreased relative to relAP2 $(-100+50)$. hns has also been reported to activate transcription from relAP2 (Nakagawa et al., 2006). However, we observed a decrease in hns mRNA levels in cells lacking 6S RNA compared with wild-type cells (A. T. Cavanagh \& K. M. Wassarman, unpublished data), suggesting that a $6 \mathrm{~S}$ RNA-dependent decrease in hns cannot account for the observed 6S RNA-dependent increase in relA expression.

\section{S RNA-dependent regulation of relA transcription leads to altered ppGpp levels}

The observed 6S RNA-dependent change in relA mRNA levels was rather modest; therefore, we next tested whether these changes in relA mRNA were sufficient to lead to a change in ppGpp levels. Wild-type and 6S RNA null (ssrS1) cells were grown in RDM containing $\left[{ }^{32} \mathrm{P}\right]$ orthophosphate. At the times indicated, nucleotides were extracted from cells by formic acid and separated by TLC (see Methods). As expected, in wild-type cells, ppGpp accumulated during the transition into stationary phase ( $4-5 \mathrm{~h}$ of growth), remained elevated for several hours in stationary phase, and then decreased to undetectable levels by $24 \mathrm{~h}$ of growth (Fig. 2) (see also Murray et al., 2003). Interestingly, in cells lacking 6S RNA, initiation of ppGpp accumulation occurred with similar timing, but the maximal level of ppGpp in early stationary phase was 1.5- to 1.6-fold higher in cells lacking 6S RNA than in wild-type cells, as also recently reported by Neusser et al. (2010). By $24 \mathrm{~h}$ of growth, ppGpp levels were undetectable in both cell types, indicating that the longer-term shut-off of ppGpp synthesis is 6S RNA-independent. ppGpp levels were undetectable in this assay throughout this time frame in $\triangle$ relA and $\Delta$ relA $\Delta$ spoT strain backgrounds (data not shown). It is

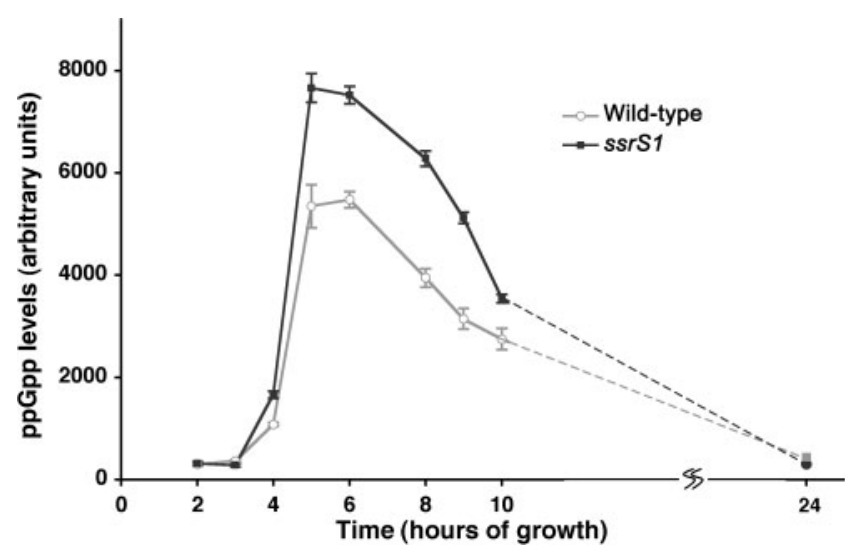

Fig. 2. Maximal ppGpp levels are higher in cells lacking 6S RNA. Nucleotides extracted by formic acid from cells grown in RDM containing $\left[{ }^{32} \mathrm{P}\right]$ orthophosphate were separated by TLC. Relative $\left.{ }^{32} \mathrm{P}\right]$ ppGpp levels are given in arbitrary intensity units as quantified on a Typhoon phosphorimager. A typical dataset with means of replicates of each cell type is shown: wild-type cells (RLG3499; lower line) and cells lacking 6S RNA (ssrS1; KW348; upper line). Error bars, SD.

clear that the observed 6S RNA-dependent downregulation of relA expression in wild-type cells is sufficient to lead to a detectable reduction in ppGpp levels in early stationary phase.

\section{RNA-dependent changes in ppGpp lead to regulation of transcription in early stationary phase}

We next tested whether the 6S RNA-dependent change in ppGpp levels might be sufficient to lead to altered transcription of promoters sensitive to ppGpp. For these experiments we chose to examine promoter-lac $Z$ reporters previously described in studies examining ppGpp and $6 \mathrm{~S}$ RNA-dependent regulation (Barker et al., 2001; Trotochaud \& Wassarman, 2004; Cavanagh et al., 2008). Note that although many of the genes examined also are regulated by attenuation or other mechanisms after transcription initiation, the reporters used here contain only minimal promoter sequences lacking elements mediating attenuation, and therefore should be representative of regulation at transcription initiation.

For promoters that are upregulated by ppGpp (i.e. livJ, hisG, thrA and pheA), expression was increased in ssrS1 compared with wild-type cells at $6 \mathrm{~h}$ of growth, a time when ppGpp levels were significantly different between cells with or without 6S RNA (Fig. 3a, Table 2). A promoter that is negatively regulated by ppGpp, $\operatorname{rrnBP}$, had decreased expression in ssrS1 compared with wild-type cells, and a ppGpp-insensitive promoter (i.e. lacUV5) was unchanged.

To further test whether these 6S RNA-dependent changes were mediated through changes in ppGpp levels, we 

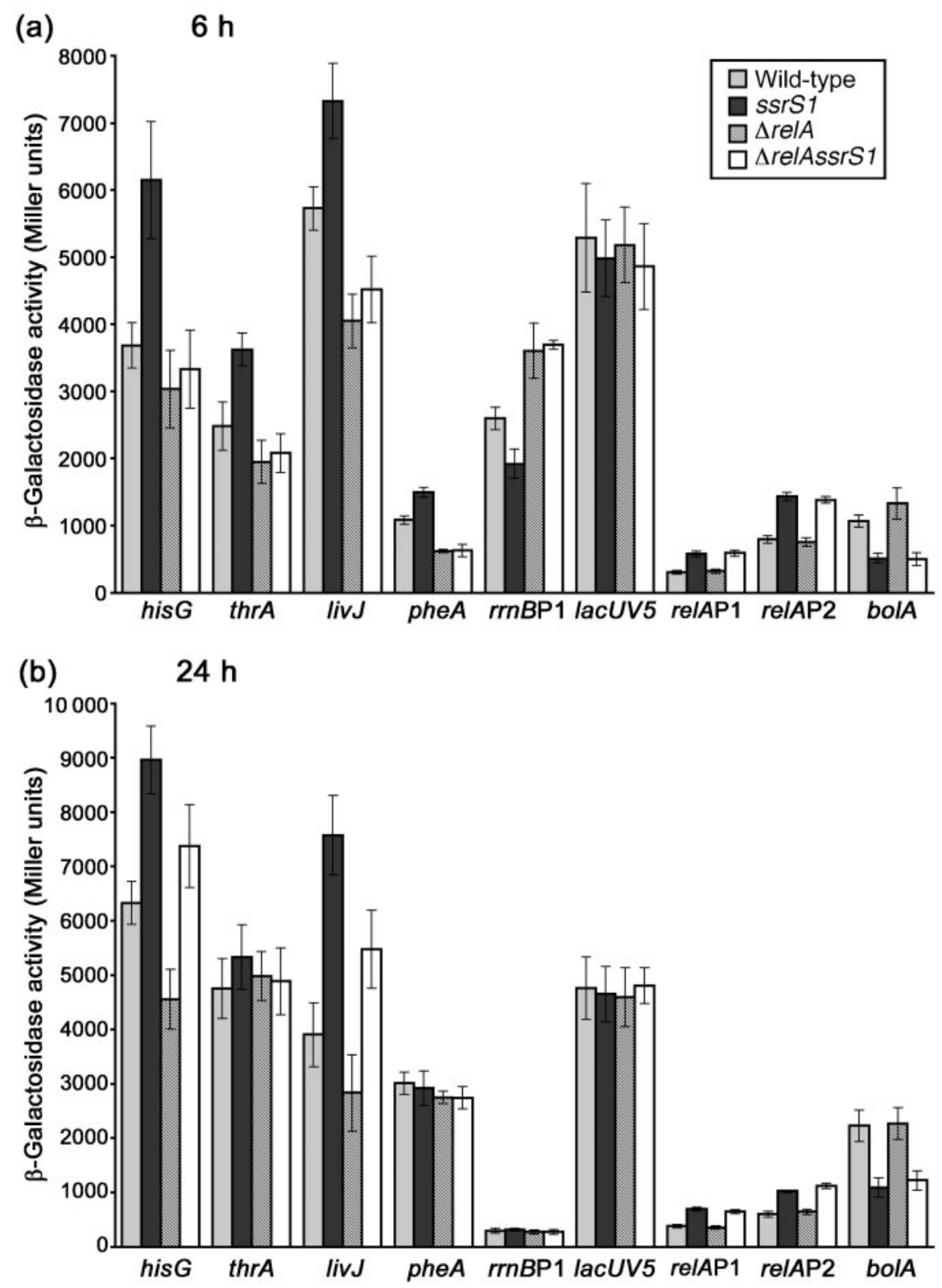

Fig. 3. $\beta$-Galactosidase activities of various promoter-lac $Z$ reporter genes in early stationary phase $(6 \mathrm{~h})$ and late stationary phase (24 h). Wild-type, 6S RNA null (ssrS1), relA null ( $\Delta$ relA) and double mutant ( $\Delta$ relAssrS1) strain backgrounds containing chromosomal copies of the promoters indicated were grown to (a) early stationary phase $(6 \mathrm{~h})$ or $(\mathrm{b})$ late stationary phase $(24 \mathrm{~h})$ in $\mathrm{RDM}$. The relA promoters are relAP1 $(-42+1)$ and relAP2 $(-41+2)$. Data shown are mean of at least three independent experiments with three duplicate samples per experiment. Error bars, SD.

measured expression of all reporters in $\Delta$ relA and $\Delta$ relAssrS1 cells (Fig. $3 \mathrm{a}$ ). Expression from the amino acid and rRNA promoters was sensitive to relA under these conditions, as expected (Barker et al., 2001); the amino acid promoters were increased in wild-type compared with $\triangle$ relA cells, and $r r n B P 1$ was decreased. However, to address whether 6S RNA-dependent changes are likely to be mediated through changes in ppGpp, the expression of reporters was compared between $\Delta$ relA and $\Delta$ relAssrS1 strain backgrounds. For hisG, thrA, livJ, pheA and rrnBP1, expression was similar in $\Delta$ relA and $\Delta$ relAssrS1 strain backgrounds, demonstrating that the $6 \mathrm{~S}$ RNA-dependent effects in early stationary phase require $r e l A$, and suggesting that they result from changes in ppGpp levels (Fig. 3a, Table 2). In contrast, expression from other $\sigma^{70}$-dependent promoters not sensitive to ppGpp [e.g. lacUV5, relAP1 $1_{(-42+2)}$ and $\left.\mathrm{relAP} 2_{(-41+2)}\right]$ and a $\sigma^{\mathrm{S}}$-dependent promoter (bolA) had the same fold changes for ssrS1 compared with the wild-type and for $\Delta$ relAssrS1 compared with $\triangle$ relA strain backgrounds (Fig. 3a, Table 2), indicating 
that not all 6S RNA-dependent changes in expression in early stationary phase require relA. Further deletion of spoT had no additional effect, as expression of the reporters tested (pheA, livJ, lacUV5 and bolA) in a $\Delta$ relA $\Delta$ spoT strain background was indistinguishable from that of the $\Delta r e l A$ background in the presence or absence of 6S RNA in early stationary phase (data not shown). Steady-state levels of $6 \mathrm{~S}$ RNA were unaffected by ppGpp, as the accumulation of $6 \mathrm{~S}$ RNA from exponential to late stationary phase in $\Delta$ relA $\triangle$ spoT cells was indistinguishable from that in wildtype cells (data not shown).

\section{RNA-dependent regulation of transcription in late stationary phase is not mediated by ppGpp}

Previously, we reported that some of the promoters examined here (i.e. livJ and hisG) were sensitive to $6 \mathrm{~S}$ RNA regulation during late stationary phase (16-24 h of growth), and at that time it was presumed to be via direct regulation (Cavanagh et al., 2008). In light of the above observations that the effects of 6S RNA on these promoters are likely to be indirect via changes in relA expression (and ppGpp levels) during early stationary phase $(6 \mathrm{~h}$ of growth), we next revisited 6S RNA-dependent changes in expression of these genes at later time frames. Of note, previous work had shown that $t h r A$, pheA and $r r n B P 1$ are not sensitive to $6 \mathrm{~S}$ RNA in late stationary phase, whereas livJ and hisG are sensitive, lending support to the suggestion that there are $6 \mathrm{~S}$ RNA effects on the transcription of the first set of genes at later times independent of those mediated through ppGpp in early stationary phase. In addition, ppGpp levels are reduced to background by $24 \mathrm{~h}$ of growth, decreasing the likelihood that it is a major regulator at these later time frames in stationary phase.

We monitored expression of our reporter genes in various strain backgrounds as indicated (e.g. with or without $6 \mathrm{~S}$ RNA, with or without relA) after 24 h of growth (Fig. 3b, Table 2). For promoters previously shown to be regulated by 6S RNA at $24 \mathrm{~h}$ (livJ, hisG), we observed that the increase in expression in the absence of 6S RNA was independent of relA at $24 \mathrm{~h}$, as indicated by a similar fold change between wild-type and ssrS1 cells and between $\Delta$ relA and $\Delta$ relAssrS1 cells for both livJ and hisG. Similarly, the $\sigma^{\mathrm{S}}$-dependent promoter bolA remained decreased in cells lacking 6S RNA in the presence or absence of relA. $6 \mathrm{~S}$ RNA regulation in late stationary phase was also not influenced by SpoT, as expression of the reporters tested (pheA, livJ, lacUV5 and bolA) in a $\Delta$ relA $\Delta$ spoT strain background was indistinguishable from that of the $\Delta r e l A$ background in the presence or absence of 6S RNA at $24 \mathrm{~h}$ of growth (data not shown). Therefore, we conclude that 6S RNA-dependent regulation of these promoters in late stationary phase is not mediated by ppGpp. In contrast, thrA, pheA, rrnBP1 and lacUV5 were insensitive to 6S RNA at $24 \mathrm{~h}$ of growth, irrespective of the presence or absence of relA.

\section{DISCUSSION}

We have demonstrated that relA transcription is a target for 6S RNA regulation, as first suggested by an expression microarray experiment and confirmed by analysis of promoter-lac $Z$ fusions. Although the changes in relA mRNA levels are modest, the changes are sufficient to direct a corresponding change in ppGpp levels. In cells lacking 6S RNA, maximum ppGpp levels are higher than in wild-type cells during early stationary phase. However, in both wild-type and 6S RNA null cells, the timing of initial ppGpp accumulation appears to be similar, and ppGpp levels are undetectable in long-term stationary phase (>24 h). In wild-type cells, ppGpp accumulates when RelA protein is activated in response to the presence of uncharged tRNAs in the ribosome, as a signal that amino acids are limiting. Later, in stationary phase, ppGpp levels decrease again, in part driven by continued degradation of ppGpp and a decrease in activation of RelA as the translational capacity of the cell is reduced in response to nutrient limitation (see Potrykus \& Cashel, 2008). We propose that 6S RNA does not alter the mechanism of activation of RelA, nor the signals and molecules involved in reducing ppGpp levels in later stationary phase. Instead, 6S RNA regulates the transcription of $\mathrm{relA}$, thereby altering the levels of RelA available to respond to these signals, and leading to changes in the levels, but not the timing, of ppGpp accumulation.

We also demonstrated that the 6S RNA-dependent changes in ppGpp during early stationary phase are sufficient to result in higher expression of genes positively regulated by ppGpp (i.e. amino acid promoters) and lower expression of a negatively regulated promoter (i.e. $\operatorname{rrn} B \mathrm{P} 1$ ). However, altered ppGpp levels cannot account for all 6S RNAdependent changes in transcription; 6S RNA regulates many promoters in late stationary phase when ppGpp levels are undetectable, and promoters that are not inherently sensitive to ppGpp also remain 6S RNAregulated in the absence of ppGpp (e.g. $\Delta$ relA strains) even during early stationary phase (see Fig. 3 ). In addition, altered ppGpp levels cannot explain the 6S RNA-dependent changes in $\sigma^{\mathrm{S}}$ activity, as demonstrated by continued $6 \mathrm{~S}$ RNA regulation of a $\sigma^{\mathrm{S}}$-dependent promoter (bolA) throughout growth in the absence of ppGpp (e.g. in $\triangle$ relA and $\Delta$ relA $\Delta$ spoT strain backgrounds). Together, these results demonstrate that 6S RNA is an additional regulator that contributes to changes in the expression of genes required to facilitate the transition from exponential to stationary phase.

\section{S RNA is required for appropriate ppGpp accumulation, but ppGpp does not influence 65 RNA activity}

There are many regulators important during the transition from exponential to stationary phase, such as ppGpp, $\sigma^{\mathrm{S}}$ and now 6S RNA. Here, we demonstrate that 6S RNA function is necessary for appropriate regulation of ppGpp 
levels during early stationary phase, raising interesting questions about whether there are additional links between these regulators. For example, given that ppGpp binds directly to $\sigma^{70}$-RNAP, one could speculate that ppGpp influences 6S RNA activity directly. However, we demonstrate here that ppGpp does not influence the ability of 6S RNA to function. Specifically, in late stationary phase, when ppGpp levels are below detection, many promoters are downregulated by 6S RNA (see Fig. 3b, hisG, livJ, relAP1 and relAP2, and additional promoters in Trotochaud \& Wassarman, 2004; Cavanagh et al., 2008), and these promoters remain similarly $6 \mathrm{~S}$ RNA-sensitive in $\triangle$ relA and $\Delta r e l A \Delta s p o T$ strain backgrounds compared with the wild-type. In early stationary phase, promoters that are not inherently sensitive to ppGpp remain sensitive to $6 \mathrm{~S}$ RNA, even in the absence of ppGpp, indicating that $6 \mathrm{~S}$ RNA function does not require ppGpp (e.g. relAP1 and relAP2; Fig. 3). In contrast, ppGpp is required for 6S RNAdependent changes in transcription from other promoters during early stationary phase (e.g. hisG, thrA, livJ, pheA and rrnBP1; see Fig. 3a). However, we suggest that these promoters are responding to changes in ppGpp levels in early stationary phase, and therefore are only indirectly regulated by $6 \mathrm{~S}$ RNA. We conclude that $6 \mathrm{~S}$ RNA activity is required for appropriate ppGpp accumulation during early stationary phase, but that ppGpp does not contribute to the ability of 6S RNA to function.

\section{S RNA-dependent changes in ppGpp levels are not responsible for $6 S$ RNA regulation of $\sigma^{s}$ activity}

We have shown previously that several $\sigma^{\mathrm{S}}$-dependent promoters are upregulated in the presence of $6 \mathrm{~S}$ RNA, although other $\sigma^{\mathrm{S}}$-dependent promoters are insensitive to 6S RNA (Trotochaud \& Wassarman, 2004), and microarray studies have suggested that expression of additional $\sigma^{\mathrm{S}}$-dependent genes is altered in cells lacking 6S RNA (Cavanagh et al., 2008; Neusser et al., 2010). We do not detect stable interactions between 6S RNA and $\sigma^{\mathrm{S}}$-RNAP in vivo or in vitro above background binding of non-specific RNAs, and steady-state levels of $\sigma^{\mathrm{S}}$ protein are unchanged in cells with and without 6S RNA (Wassarman \& Storz, 2000; Trotochaud \& Wassarman, 2005). Therefore, we proposed that 6S RNA-dependent regulation of $\sigma^{\mathrm{S}}$ activity is indirect. Given that ppGpp can increase alternative $\sigma$ factor activity by a number of proposed mechanisms (Jishage et al., 2002; Bernardo et al., 2006; Costanzo et al., 2008), we wondered whether the 6S RNA upregulation of $\sigma^{\mathrm{S}}$-dependent promoters is mediated through changes in ppGpp. However, we found that a $\sigma^{\mathrm{S}}$-dependent promoter previously shown to be sensitive to 6S RNA (bolA) (Trotochaud \& Wassarman, 2004) remained similarly sensitive to 6S RNA in both early and late stationary phase in $\triangle$ relA and $\triangle$ relA $\triangle$ spoT strain backgrounds compared with the wild-type (Fig. 3, Table 2; data not shown). In addition, previous studies on $\sigma^{\mathrm{S}}$-dependent promoters were carried out in late stationary phase, when ppGpp levels are undetectable (Trotochaud \& Wassarman, 2004). Although it is possible that some $\sigma^{\mathrm{S}}$-promoters will respond to the $6 \mathrm{~S}$ RNA-dependent changes in ppGpp levels, these data demonstrate that 6S RNA upregulation of $\sigma^{\mathrm{S}}$-dependent transcription cannot simply be explained by the changes in ppGpp levels. Further studies will be needed to explain how 6S RNA function leads to altered $\sigma^{\mathrm{S}}$-dependent transcription.

\section{S RNA regulation of transcription: direct versus indirect mechanisms}

As we aim to elucidate the mechanisms by which 6S RNA regulates transcription and the cellular consequences of this regulation, it becomes increasingly important to try to separate direct and indirect effects. Here, we have clearly shown that in early stationary phase, 6S RNA-dependent changes in expression of amino acid promoters and rRNA promoters are indirect, and result from altered relA expression. However, we propose that the 6S RNAdependent change in relA expression is due, at least in part, to direct regulation (see below). Typically, direct transcriptional regulation would be verified using purified in vitro transcription assays. Unfortunately, this approach has not proven useful in studies of 6S RNA to date; the in vitro transcription conditions tested have not recapitulated promoter-specific regulation by 6S RNA, but instead all tested $\sigma^{70}$-dependent promoters have been similarly downregulated (Trotochaud \& Wassarman, 2005; Wassarman \& Saecker, 2006). It is possible that other factors involved in 6S RNA function in vivo are lacking in the in vitro assay, or that the in vitro conditions are not dynamic enough to represent in vivo conditions. We favour the second possibility, based on observations that 6S RNA and promoter DNA bound to $\sigma^{70}$-RNAP have very slow offrates in vitro, suggesting that the tested in vitro conditions are not able to effectively reproduce the presumed competition occurring in vivo (Wassarman \& Saecker, 2006; Klocko \& Wassarman, 2009). However, we note that 6S RNA regulation of $\sigma^{70}$-dependent transcription does not result from competition with $\sigma^{\mathrm{s}}$, as tested $\sigma^{70}$-dependent promoters (e.g. $r s d \mathrm{P} 2$; see Trotochaud \& Wassarman, 2004) remain 6S RNA-sensitive in an rpoS:: Tn 10 strain background (data not shown).

The inability to recapitulate the promoter specificity for $6 \mathrm{~S}$ RNA regulation using in vitro assays has made definitive testing of direct versus indirect 6S RNA effects more difficult. However, we previously identified two promoter features that correlated with 6S RNA regulation (a weak -35 element and an extended -10 element) using reporters with minimal promoter sequences that lacked known binding sites for trans-acting factors to minimize the potential for indirect effects. More importantly, we were able to convert sensitive to insensitive promoters as well as insensitive to sensitive promoters with minor sequence changes only in core promoter elements of these reporters, even in several unrelated promoters that do not 
share flanking sequences (e.g. livJ, lacUV5, hupBP2, $p s p F$ and galP2) (Cavanagh et al., 2008). These results strongly support the model that these elements are important for direct $6 \mathrm{~S}$ RNA regulation, presumably through competition between 6S RNA and promoter DNA for binding to $\sigma^{70}$-RNAP.

Based on our previous study, we predicted that 6S RNAdependent regulation of relA is likely to be direct at both P1 and $\mathrm{P} 2$, which have a weak -35 element and an extended -10 element, respectively. Both minimal promoter fusions were 6S RNA-sensitive, consistent with our hypothesis (see Fig. 1c, d). However, we also note that relAP2 $(-100+50)$ is more sensitive to $6 \mathrm{~S}$ RNA than relAP2 ${ }_{(-41+2)}$, suggesting that there are additional effects at the longer promoter, most likely mediated through the CRP binding site and $6 \mathrm{~S}$ RNA-dependent changes in crp expression (see Fig. 1c, e). Results from a microarray experiment comparing global mRNA levels in cells with and without 6S RNA revealed that $\operatorname{crp}$ mRNA levels were increased 1.9-fold in cells lacking 6S RNA. Transcription of $c r p$ is complex, with multiple promoters, making it more difficult to predict whether it is directly regulated by 6S RNA without further experimentation; however, we note that one of the $\mathrm{crp}$ promoters contains an extended -10 element (Mitchell et al., 2003), suggesting that it is a possibility.

Intriguingly, we have been able to distinguish two mechanisms by which 6S RNA regulates expression from some amino acid biosynthesis and uptake genes: (1) an indirect mechanism in early stationary phase $(6 \mathrm{~h})$, in which 6S RNA-dependent changes in ppGpp levels lead to changes in transcription at all tested ppGpp-sensitive promoters; and (2) a relA-independent mechanism in late stationary phase $(\geqslant 24 \mathrm{~h})$, in which $6 \mathrm{~S}$ RNA-dependent changes in transcription are not mediated through changes in levels of ppGpp. We maintain our previous predictions that the late stationary phase regulation by $6 \mathrm{~S}$ RNA is direct, based on the presence of weak -35 elements in the sensitive promoters ( $h i s G$ and livJ), the observation that relA is dispensable at this time (see Fig. 3b, Table 2), and the ability to convert the livJ reporter to a 6S RNAinsensitive promoter by changing the -35 element to consensus (Cavanagh et al., 2008). However, it remains possible that the observed changes at these promoters are mediated through another gene with altered expression, analogous to the role of relA with respect to ppGppsensitive promoters in early stationary phase and of $c r p$ with respect to relAP2.

\section{The role of 65 RNA in stationary phase}

Regardless of whether we can fully distinguish direct from indirect $6 \mathrm{~S}$ RNA regulation of transcription, we can gain insight into how the function of 6S RNA affects cellular physiology by considering the global changes in gene expression, as all changes (direct and indirect) will contribute. We previously reported that hundreds of genes are altered in expression in late stationary phase in a $6 \mathrm{~S}$
RNA-dependent manner (Cavanagh et al., 2008), and recently others have reported large numbers of changes in earlier time frames as well (Neusser et al., 2010). The two studies used microarray analysis to compare global gene expression changes in genes with and without 6S RNA, and therefore changes in mRNA levels represent both 6S RNAdirect and 6S RNA-indirect changes. As such, it would be surprising to find signals important for direct 6S RNA regulation to be present in all genes altered in expression, nor should it be unexpected that some genes have decreased expression in the absence of 6S RNA, even among those with $\sigma^{70}$-dependent promoters. Here, we set out to test whether there was a relationship between $6 \mathrm{~S}$ RNA and ppGpp, as both are regulators of transcription during stationary phase; therefore, we focused on several well-studied promoters known to be sensitive to ppGpp (e.g. amino acid promoters and $r r n \mathrm{BP} 1$ ). Our findings are consistent with a recent report that also observed 6S RNAdependent changes in ppGpp levels and focused primarily on genes encoding the translation machinery that are sensitive to ppGpp (Neusser et al., 2010).

It is intriguing to speculate why one stationary phase regulator, 6S RNA, downregulates another regulator, relA, that is important during the transition into stationary phase. The timing of ppGpp accumulation is not dramatically altered in cells lacking 6S RNA compared with wild-type cells; instead, it appears to be the maximal level of ppGpp accumulation that is changed (see Fig. 2). One model suggests that the role of $6 \mathrm{~S}$ RNA in stationary phase is to dampen the expression of many genes, perhaps to conserve energy as cells encounter suboptimal growth conditions (Trotochaud \& Wassarman, 2006). As such, 6S RNA would facilitate the integration of multiple signal inputs by downregulating key regulators in multiple pathways and preventing overactivation of any one response. For example, one gene downregulated by $6 \mathrm{~S}$ RNA, $p s p F$, is a transcriptional activator that responds to several stresses, including elevated pH (Model et al., 1997; Darwin, 2005). We have shown that the presence of $6 \mathrm{~S}$ RNA decreases the set-point of $p s p F$ expression at neutral $\mathrm{pH}$ but does not alter the ability of the psp system to respond to high $\mathrm{pH}$ conditions (Trotochaud \& Wassarman, 2006). Many key regulators appear to be differentially expressed in cells lacking 6S RNA compared with wild-type cells, such as CRP (see above) and OxyR (A. T. Cavanagh \& K. M. Wassarman, unpublished data), suggesting that several diverse pathways are affected by 6S RNA function, and that understanding the full extent of how 6S RNA function alters cell physiology will require further studies to unravel these complexities.

\section{ACKNOWLEDGEMENTS}

We thank R. Gourse, W. Ross and T. Gaal for helpful discussions throughout this work, and Jamie Sperger for critical reading of the manuscript. We thank R. Gourse, University of Wisconsin-Madison, for strains, and T. Gaal and C. Vrentas for advice on ppGpp analysis. This work was supported by the National Institutes of Health (GM67955). 


\section{REFERENCES}

Barker, M. M., Gaal, T., Josaitis, C. A. \& Gourse, R. L. (2001). Mechanism of regulation of transcription initiation by ppGpp. I. Effects of ppGpp on transcription initiation in vivo and in vitro. $\mathrm{J} \mathrm{Mol}$ Biol 305, 673-688.

Bernardo, L. M., Johansson, L. U., Solera, D., Skärfstad, E. \& Shingler, V. (2006). The guanosine tetraphosphate (ppGpp) alarmone, DksA and promoter affinity for RNA polymerase in regulation of $\sigma^{54}$-dependent transcription. Mol Microbiol 60, 749-764.

Bown, J. A., Barne, K. A., Minchin, S. D. \& Busby, S. J. W. (1997). Extended -10 promoters. Nucleic Acids Mol Biol 11, 41-52.

Cavanagh, A. T., Klocko, A. D., Liu, X. \& Wassarman, K. M. (2008). Promoter specificity for 6S RNA regulation of transcription is determined by core promoter sequences and competition for region 4.2 of $\sigma^{70}$. Mol Microbiol 67, 1242-1256.

Costanzo, A., Nicoloff, H., Barchinger, S. E., Banta, A. B., Gourse, R. L. \& Ades, S. E. (2008). ppGpp and DksA likely regulate the activity of the extracytoplasmic stress factor $\sigma^{\mathrm{E}}$ in Escherichia coli by both direct and indirect mechanisms. Mol Microbiol 67, 619-632.

Darwin, A. J. (2005). The phage-shock-protein response. Mol Microbiol 57, 621-628.

Gaal, T., Ross, W., Estrem, S. T., Nguyen, L. H., Burgess, R. R. \& Gourse, R. L. (2001). Promoter recognition and discrimination by $\mathrm{E} \sigma^{\mathrm{S}}$ RNA polymerase. Mol Microbiol 42, 939-954.

Gralla, J. D. (2005). Escherichia coli ribosomal RNA transcription: regulatory roles for ppGpp, NTPs, architectural proteins and a polymerase-binding protein. Mol Microbiol 55, 973-977.

Hindley, J. (1967). Fractionation of ${ }^{32} \mathrm{P}$-labelled ribonucleic acids on polyacrylamide gels and their characterization by fingerprinting. $J \mathrm{Mol}$ Biol 30, 125-136.

Hirvonen, C. A., Ross, W., Wozniak, C. E., Marasco, E., Anthony, J. R., Aiyar, S. E., Newburn, V. H. \& Gourse, R. L. (2001). Contributions of UP elements and the transcription factor FIS to expression from the seven rrn P1 promoters in Escherichia coli. J Bacteriol 183, 6305-6314.

Jishage, M., Kvint, K., Shingler, V. \& Nyström, T. (2002). Regulation of sigma factor competition by the alarmone ppGpp. Genes Dev 16, $1260-1270$.

Klocko, A. D. \& Wassarman, K. M. (2009). $6 \mathrm{~S}$ RNA binding to $\mathrm{E} \sigma^{70}$ requires a positively charged surface of $\sigma^{70}$ region 4.2. Mol Microbiol 73, 152-164.

Lee, C. A., Fournier, M. J. \& Beckwith, J. (1985). Escherichia coli $6 S$ RNA is not essential for growth or protein secretion. J Bacteriol 161, 1156-1161.

Magnusson, L. U., Farewell, A. \& Nyström, T. (2005). ppGpp: a global regulator in Escherichia coli. Trends Microbiol 13, 236-242.

Metzger, S., Dror, I. B., Aizenman, E., Schreiber, G., Toone, M., Friesen, J. D., Cashel, M. \& Glaser, G. (1988). The nucleotide sequence and characterization of the relA gene of Escherichia coli. J Biol Chem 263, 15699-15704.

Miller, J. H. (1972). Experiments in Molecular Genetics. Cold Spring Harbor, NY: Cold Spring Harbor Laboratory.

Mitchell, J. E., Zheng, D., Busby, S. J. W. \& Minchin, S. D. (2003). Identification and analysis of "extended -10 " promoters in Escherichia coli. Nucleic Acids Res 31, 4689-4695.

Model, P., Jovanovic, G. \& Dworkin, J. (1997). The Escherichia coli phage-shock-protein ( $p s p$ ) operon. Mol Microbiol 24, 255-261.
Murray, H. D., Schneider, D. A. \& Gourse, R. L. (2003). Control of rRNA expression by small molecules is dynamic and nonredundant. Mol Cell 12, 125-134.

Nakagawa, A., Oshima, T. \& Mori, H. (2006). Identification and characterization of a second, inducible promoter of relA in Escherichia coli. Genes Genet Syst 81, 299-310.

Neidhardt, F. C., Bloch, P. L. \& Smith, D. F. (1974). Culture medium for enterobacteria. $J$ Bacteriol 119, 736-747.

Neusser, T., Polen, T., Geissen, R. \& Wagner, R. (2010). Depletion of the non-coding regulatory $6 \mathrm{~S}$ RNA in E. coli causes a surprising reduction in the expression of the translation machinery. $B M C$ Genomics 11, 165-178.

Paul, B. J., Ross, W., Gaal, T. \& Gourse, R. L. (2004). rRNA transcription in Escherichia coli. Annu Rev Genet 38, 749-770.

Potrykus, K. \& Cashel, M. (2008). (p)ppGpp: still magical? Annu Rev Microbiol 62, 35-51.

Rao, L., Ross, W., Appleman, J. A., Gaal, T., Leirmo, S., Schlax, P. J., Record, M. T., Jr \& Gourse, R. L. (1994). Factor independent activation of $r r n B P 1$. An "extended" promoter with an upstream element that dramatically increases promoter strength. J Mol Biol 235, 1421-1435.

Schneider, D. A. \& Gourse, R. L. (2004). Relationship between growth rate and ATP concentration in Escherichia coli: a bioassay for available cellular ATP. J Biol Chem 279, 8262-8268.

Schneider, D. A., Murray, H. D. \& Gourse, R. L. (2003). Measuring control of transcription initiation by changing concentrations of nucleotides and their derivatives. Methods Enzymol 370, 606-617.

Silhavy, T. J., Berman, M. L. \& Enquist, L. W. (1984). Experiments with Gene Fusions. Cold Spring Harbor, NY: Cold Spring Harbor Laboratory.

Srivatsan, A. \& Wang, J. D. (2008). Control of bacterial transcription, translation and replication by (p)ppGpp. Curr Opin Microbiol 11, 100-105.

Trotochaud, A. E. \& Wassarman, K. M. (2004). 6S RNA function enhances long-term cell survival. J Bacteriol 186, 4978-4985.

Trotochaud, A. E. \& Wassarman, K. M. (2005). A highly conserved 6S RNA structure is required for regulation of transcription. Nat Struct Mol Biol 12, 313-319.

Trotochaud, A. E. \& Wassarman, K. M. (2006). 6S RNA regulation of $p s p F$ transcription leads to altered cell survival at high $\mathrm{pH}$. J Bacteriol 188, 3936-3943.

Voskuil, M. I., Voepel, K. \& Chambliss, G. H. (1995). The -16 region, a vital sequence for the utilization of a promoter in Bacillus subtilis and Escherichia coli. Mol Microbiol 17, 271-279.

Wassarman, K. M. (2007). 6S RNA: a regulator of transcription. Mol Microbiol 65, 1425-1431.

Wassarman, K. M. \& Saecker, R. M. (2006). Synthesis-mediated release of a small RNA inhibitor of RNA polymerase. Science 314, 1601-1603.

Wassarman, K. M. \& Storz, G. (2000). 6S RNA regulates E. coli RNA polymerase activity. Cell 101, 613-623.

Willkomm, D. K. \& Hartmann, R. K. (2005). 6S RNA - an ancient regulator of bacterial RNA polymerase rediscovered. Biol Chem 386, 1273-1277.

Xiao, H., Kalman, M., Ikehara, K., Zemel, S., Glaser, G. \& Cashel, M. (1991). Residual guanosine $3^{\prime}, 5^{\prime}$-bispyrophosphate synthetic activity of relA null mutants can be eliminated by spoT null mutations. J Biol Chem 266, 5980-5990.

Edited by: J.-H. Roe 